\title{
Nursing intervention using a whole-process escort playing a relative role combined with mind mapping in patients undergoing breast cancer surgery: a randomized trial
}

\author{
Li Fan ${ }^{1}$, Fengqiong Lei $^{2}$, Zhen Zhu ${ }^{1}$, Chaoqun $\mathrm{Hu}^{1}$, Lei Ye ${ }^{1}$, Na Wang ${ }^{3}$ \\ ${ }^{1}$ Operating Room, Wuhan Children's Hospital (Wuhan Maternal and Child Healthcare Hospital), Tongji Medical College, Huazhong University of \\ Science \& Technology, Wuhan, China; ${ }^{2}$ Nursing Department, Wuhan Children's Hospital (Wuhan Maternal and Child Healthcare Hospital), Tongji \\ Medical College, Huazhong University of Science \& Technology, Wuhan, China; ${ }^{3}$ Nursing Department, Wuhan Wuchang Hospital, Wuhan, China \\ Contributions: (I) Conception and design: L Fan; (II) Administrative support: Z Zhu; (III) Provision of study materials or patients: C Hu, F Lei; (IV) \\ Collection and assembly of data: L Fan, F Lei; (V) Data analysis and interpretation: C Hu; (VI) Manuscript writing: All authors; (VII) Final approval \\ of manuscript: All authors. \\ Correspondence to: Li Fan. Wuhan Children's Hospital (Wuhan Maternal and Child Healthcare Hospital), Tongji Medical College, Huazhong \\ University of Science \& Technology, Wuhan, China. Email: fanli_m@sina.com.
}

\begin{abstract}
Background Breast cancer is the most common malignant tumor affecting women worldwide. Surgery is the main treatment for breast cancer and can obtain a good curative effect. However, it can also entail trauma and stress for patients, leaving them susceptible to negative emotions, poor sleep quality, and various postoperative complications, which can affect the treatment effect and prognosis. Therefore, it is of great significance to explore scientific and effective nursing methods for patients with breast cancer. This study aimed to examine the application effect of whole-process nursing and mind mapping in patients with breast cancer.
\end{abstract}

Methods: The present study enrolled 256 patients with breast cancer who were admitted to the hospital from March 2019 to October 2020. The study participants were divided into a control group (routine nursing intervention) and an observation group (nursing intervention including a whole-process escort playing a relative role combined with mind mapping) according to the random number table method, with 128 cases in each group. At admission and before surgery, the psychological status [Hamilton anxiety scale (HAMA) and Hamilton depression scale (HAMD)], physiological stress [cortisol (Cs) and adrenocorticotrophic hormone (ACTH)], fatigue degree (Piper fatigue scale), and sleep quality [Pittsburgh sleep quality index (PSQI)] were compared between the two groups, and the incidence of complications in the two groups was also compared. Results: The psychological status (HAMA and HAMD) scores, physiological stress levels (Cs and ACTH), fatigue degree (Piper fatigue scale), and sleep quality (PSQI) score were all significantly higher before surgery than at admission in both groups, and were significantly lower in the observation group than in the control group $(\mathrm{P}<0.05)$. There were no significant differences in the incidence of complications between the two groups $(\mathrm{P}>0.05)$.

Conclusions: Nursing intervention using a whole-process escort playing a relative role combined with mind mapping can effectively relieve the anxiety and depression felt by patients undergoing breast cancer surgery, and can reduce their physiological stress and fatigue degree, as well as improve their sleep quality.

Trial Registration: Chinese Clinical Trial Registry ChiCTR2100054024.

Keywords: Playing a relative role; whole-process escort; mind mapping; breast cancer; surgery

Submitted Feb 24, 2021. Accepted for publication Dec 17, 2021.

doi: 10.21037/apm-21-915

View this article at: https://dx.doi.org/10.21037/apm-21-915 


\section{Introduction}

Breast cancer is the most common malignant tumor affecting women, the incidence and prevalence rate of which are increasing globally (1). Owing to the development and progress of medical technology in recent years, some patients with breast cancer can be screened out and treated at an early stage, which can significantly prolong patients' survival time and preserve their quality of life (2). Surgery is the main treatment for breast cancer and can obtain a good curative effect. However, surgery also results in trauma and stress for patients, leaving them prone to a variety of postoperative complications, which can affect the treatment effect and prognosis (3). The "playing a relative role" method is a psychological intervention in which medical staff dress up as patients' families and accompany patients for treatment so as to alleviate the negative emotions felt by patients (4). Mind mapping, also known as brain mapping, is a technique which combines pictures and text to summarize information and convert it into graphics to facilitate understanding and memory, with the aim of improving thinking efficiency (5). Mind mapping can encourage the brain to think according to its own laws, mobilize the brain's collaborative work, and facilitate better choices and problem solving (6). In the present study, we combined the assignment of a whole-process escort playing a relative role with mind mapping and explored the effect of this approach in patients undergoing breast cancer surgery.

We present the following article in accordance with the CONSORT reporting checklist (available at https://dx.doi. org/10.21037/apm-21-915).

\section{Methods}

\section{General information}

This study is designed as a random parallel study, and allocation ratio is $1: 1$. A total of 256 patients who received treatment for breast cancer in Wuhan Children's Hospital (Wuhan Maternal and Child Healthcare Hospital) between March 2019 and October 2020 were randomly divided into a control group $(n=128)$ and an observation group $(n=128)$ according to the random number table method (Figure 1). The participant inclusion criteria were: (I) Patients with breast cancer diagnosed by pathological examination and scheduled to undergo mastectomy; and (II) aged $\geq 18$ years old. Participants' informed consent was obtained via a signed form.

Participant exclusion criteria included: (I) patients who were unconscious or unable to communicate; (II) critically ill patients with advanced breast cancer with distant metastasis and multiple organ failure; (III) patients with dysfunction of the heart, lung, kidney, or another important organ; (IV) patients with mental disorders.

The study was approved by ethics board of Wuhan Children's Hospital (Wuhan Maternal and Child Healthcare Hospital, No. 2021R55-E01). All procedures performed in this study involving human participants were in accordance with the Declaration of Helsinki (as revised in 2013).

\section{Study methods}

\section{Interventional methods}

The control group was treated with routine nursing intervention. During treatment, the nursing staff observed and recorded patients' postoperative vital signs, and conducted health education, including in relation to medication and matters requiring attention after the operation. The observation group intervention included the assignment of a whole-process escort playing a relative role combined with mind mapping. The main contents of the nursing intervention in the observation group are as follows.

\section{Whole-process escort playing a relative role}

(I) Establishing the patient-nurse relationship: the nurses talked actively with patients who were about to undergo breast cancer surgery after their admission to hospital to gain an understanding of their psychological state, family environment, social relations, economic status, and cognition of breast cancer. The nurses established a good relationship with patients and addressed them as relatives. (II) Health education: the nurses explained the operating room environment and principle of the operation to the patients to ensure the patients had a certain understanding of their breast cancer surgery. The nurses encouraged the patients to ask questions, and gave meticulous and comprehensive answers to familiarize the patients with the instruments and equipment which would be used during the operation. Furthermore, the nurses introduced the surgical medical staff to the patient to improve their familiarity. (III) Psychological nursing: the nurses accompanied the patients throughout the entire process, acting as relatives, and provided timely targeted guidance when patients were under psychological pressure, such as diverting their attention, talking, etc., to guide patients to meditate and have relaxed and happy thoughts. The nurses also accompanied the patients during the operation and assisted patients in getting through the operation smoothly. 


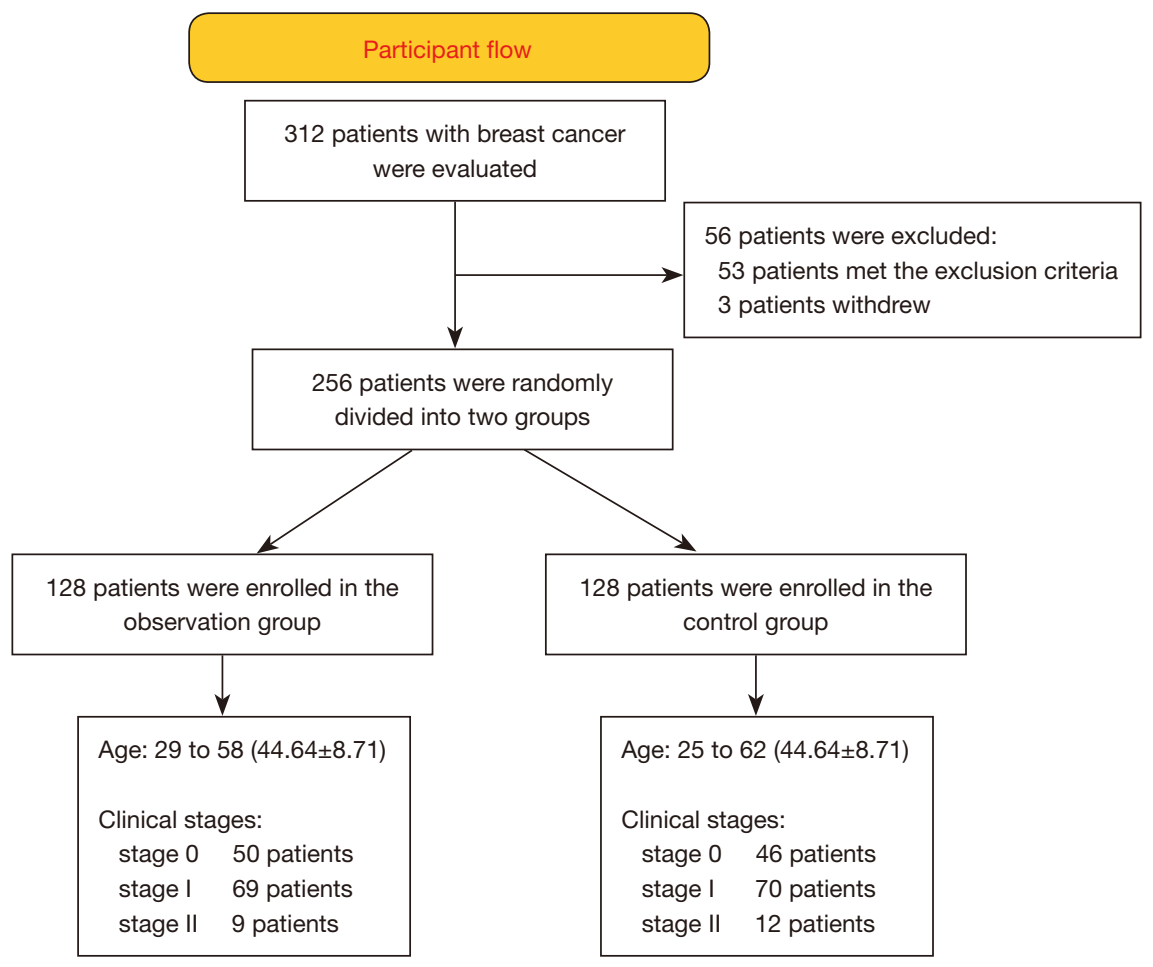

Figure 1 Flow chart of patients in this study.

\section{Mind mapping}

(I) Designing the mind map: the mind map was divided into 3 second-level branches (before, during and after surgery), centering on the perioperative nursing of breast cancer patients. The map included: 3 preoperative third-level branches: admission guidance, preoperative preparation, and psychological nursing; 2 perioperative third-level branches: warmth and psychological nursing; and 4 postoperative thirdlevel branches: nursing methods for bleeding, subcutaneous effusion, upper limb swelling, and skin flap necrosis. After completing the design, we make a tree view, used bright colors and pictures, and used the mind-mapping software Mind Manager to draw the map, which was printed on paper, covered the outside with plastic for long-term use. (II) Implementation of mind mapping: The nurses were trained in mind mapping, which required them to fully understand and remember the mind mapping, and to carry out clinical nursing work according to the mind map. (III) Follow-up investigation: We regularly asked all the nurses to retrospectively analyze any problems in the implementation of the mind map. With the combination of literature and clinical nursing experience, we gradually improved the mind map to make the nursing work plan more perfect.

\section{Index detection method}

Fasting venous blood (5 mL) was collected from the participants at admission and before surgery, and plasma was collected by centrifugation. The levels of cortisol (cortisol, Cs) and adrenocorticotropic hormone (ACTH) were determined by radioimmunoassay.

\section{Evaluation criteria}

\section{Mental state}

The Hamilton anxiety scale (HAMA) (7), which comprises 14 items, was used to assess the participants' anxiety. Using the scale, each item is given a score of 0 to 4 points, with the total score ranging from 0 to 56 points; the higher a patient's score, the more severe their anxiety. The Hamilton depression scale (HAMD) (8), which includes 17 items, was used to assess the participants' depression. Each item in the scale is scored from 0 to 4 points, with a total score range of 0 to 68 points; the higher a patient's score, the more severe their depression.

\section{Fatigue degree}

The Piper fatigue scale (9) was used to evaluate the degree 
Table 1 Comparison of HAMA and HAMD levels between the 2 groups $(\bar{x} \pm \mathrm{s})$

\begin{tabular}{lllll}
\hline Group & No. & Time & HAMA & HAMD \\
\hline Observation group & 128 & At admission & $12.26 \pm 2.34$ & $14.10 \pm 1.95$ \\
& & Before surgery & $14.71 \pm 2.13^{\star \Delta}$ & $16.28 \pm 2.42^{\star \Delta}$ \\
Control group & 128 & At admission & $12.51 \pm 2.29$ & $14.07 \pm 2.18$ \\
& & Before surgery & $16.24 \pm 2.32^{\star}$ & $19.14 \pm 2.51^{*}$ \\
\hline
\end{tabular}

*, compared with the same group at admission, $\mathrm{P}<0.05 ;{ }^{\wedge}$, compared with the control group, $\mathrm{P}<0.05$. HAMA, Hamilton anxiety scale; HAMD, Hamilton depression scale.

of cancer-related fatigue. The scale includes 5 dimensions: behavior, emotion, feeling, cognition, and station. The higher the score, the more severe the fatigue.

\section{Sleep quality}

The Pittsburgh sleep quality index (PSQI) scale (10) was used to evaluate the participants' sleep quality. The scale comprises 24 items, each of which is given a score of 0 to 3 points, with a total score range of 0 to 21 points; the higher the score, the poorer the sleep quality.

\section{Observation index}

The mental state (HAMA and HAMD scales), physiological stress (Cs and ACTH levels), fatigue degree (Piper fatigue scale), and sleep quality (PSQI) were compared between the observation and control groups at admission and before surgery.

\section{Statistical methods}

Data were analyzed using SPSS22.0 software (International Business Machines Corporation, United States). Counting data were expressed by rate (\%), comparisons between groups were performed using the $\chi^{2}$ test. Measurement data were expressed by $\left(\bar{x}_{ \pm} \leq\right)$, and the independent-samples $t$-test was used for comparisons between groups. $\mathrm{P}<0.05$ indicated a significant difference between the 2 groups.

\section{Results}

\section{General information of patients in two groups}

The participants in the observation group ranged in age from 29 to 58 years old, with the average age being $(44.64 \pm 8.71)$ years. In terms of clinical stages, 50, 69, and 9 patients in the observation group were stage 0 , I, and II, respectively. The ages of the control group participants ranged from 25 to 62 years old, with the average age being $(45.85 \pm 8.95)$ years. In the control group, 46, 70, and 12 participants had clinical stage 0 , I, and II, respectively (Figure 1). All patients were evaluated during after admission and before operation. General data, such as age and clinical stage, were balanced and comparable between the 2 groups $(\mathrm{P}>0.05)$.

\section{Comparison of mental state between the 2 groups}

In both groups, the HAMA and HAMD scores were significantly higher before surgery than at admission. However, the HAMA and HAMD scores in the observation group were significantly lower than those in the control group $(\mathrm{P}<0.05)$ (Table 1$)$.

\section{Comparison of physiological stress between the 2 groups}

The levels of Cs and ACTH were significantly higher before surgery than at admission in both groups. However, the levels in the observation group were significantly lower than those in the control group $(\mathrm{P}<0.05)$ (Table 2$)$.

\section{Comparison of fatigue degree between the 2 groups}

In both groups, the Piper fatigue scale scores were significantly higher before the operation than at admission. However, the Piper Fatigue Scale score in the observation group was significantly lower than that in the control group $(\mathrm{P}<0.05)$ (Table 3).

\section{Comparison of sleep quality between the 2 groups}

The PSQI scores were significantly higher before surgery than at admission in both groups. However, the PSQI score in the observation group was significantly lower than that in 
Table 2 Comparison of $\mathrm{Cs}$ and ACTH levels between the 2 groups $(\bar{x} \pm \mathrm{s})$

\begin{tabular}{lcccc}
\hline Group & No. & Time & Cs $(\mathrm{nmol} / \mathrm{L})$ & ACTH $(\mathrm{pg} / \mathrm{mL})$ \\
\hline Observation group & 128 & At admission & $373.32 \pm 65.81$ & $24.90 \pm 4.45$ \\
& & Before surgery & $425.75 \pm 60.62^{\star \Delta}$ & $31.08 \pm 5.87^{\star \Delta}$ \\
Control group & 128 & At admission & $373.51 \pm 68.58$ & $25.27 \pm 4.38$ \\
& & Before surgery & $468.14 \pm 52.91^{\star}$ & $37.34 \pm 6.51^{\star}$ \\
\hline
\end{tabular}

*, compared with the same group at admission, $\mathrm{P}<0.05 ;{ }^{\Delta}$, compared with the control group, $\mathrm{P}<0.05$. Cs, cortisol; ACTH, adrenocorticotrophic hormone.

Table 3 Comparison of piper fatigue scale scores between the 2 groups $(\bar{x} \pm \mathrm{s})$

\begin{tabular}{lccccccc}
\hline Group & No. & Time & Overall evaluation & Behavior & Emotion & Feeling & Cognition and station \\
\hline Observation group & 128 & At admission & $4.16 \pm 0.75$ & $4.13 \pm 0.73$ & $4.11 \pm 0.59$ & $4.14 \pm 0.58$ & $4.01 \pm 0.54$ \\
& & Before surgery & $4.79 \pm 0.73^{\star \Delta}$ & $4.81 \pm 0.64^{\star \Delta}$ & $4.81 \pm 0.53^{\star \Delta}$ & $4.60 \pm 0.54^{\star \Delta}$ & $4.66 \pm 0.49^{* \Delta}$ \\
Control group & 128 & At admission & $4.11 \pm 0.79$ & $4.11 \pm 0.76$ & $4.10 \pm 0.54$ & $4.10 \pm 0.53$ & $4.05 \pm 0.50$ \\
& & Before surgery & $5.30 \pm 1.02^{*}$ & $5.49 \pm 0.57^{*}$ & $5.46 \pm 0.70^{*}$ & $5.29 \pm 0.59^{*}$ & $5.21 \pm 0.69^{*}$ \\
\hline
\end{tabular}

*, compared with the same group at admission, $\mathrm{P}<0.05{ }^{\Delta}$, compared with the control group, $\mathrm{P}<0.05$.

Table 4 Comparison of PSQI scores between the 2 groups $(x \pm s)$

\begin{tabular}{lccc}
\hline Group & No. & Time & PSQI \\
\hline Observation group & 128 & At admission & $10.17 \pm 2.21$ \\
& & Before surgery & $12.44 \pm 2.08^{\star \Delta}$ \\
Control group & 128 & At admission & $10.24 \pm 2.10$ \\
& & Before surgery & $14.21 \pm 2.35^{\star}$ \\
\hline
\end{tabular}

*, compared with the same group at admission, $\mathrm{P}<0.05 ;{ }^{\Delta}$, compared with the control group, $\mathrm{P}<0.05$. PSQ, Pittsburgh sleep quality index.

the control group $(\mathrm{P}<0.05)$ (Table 4).

\section{Comparison of complications between the 2 groups}

No significant difference was observed in the incidence of complications between the 2 groups $(\mathrm{P}>0.05)$ (Table 5).

\section{Discussion}

Breast cancer is a malignant tumor with a high incidence among women worldwide. Moreover, with the changes in social environment, the incidence of breast cancer is increasing year by year (11). The exploration of breast cancer treatment can significantly prolong the survival time of patients, and mastectomy can effectively treat the disease. However, patients often have concerns about the effects of surgery and their safety. Together with the fear of disease and death, and psychological depression and pain, the weight of these concerns increases the risk of patients experiencing negative moods and poor sleep quality (12). A negative mood can impact the occurrence and development of malignant tumors, as well as patients' treatment and prognosis, and may result in a vicious cycle. Consequently, it is of great significance to explore scientific and effective nursing methods for patients with breast cancer.

The "playing a relative role" method is an intervention in which nurses, acting as relatives of patients, encourage patients to express their true thoughts and listen to their inner feelings, with a caring attitude. As a result, patients can vent their negative emotions, and nurses can understand patients' cognition of diseases and treatments. Thus, the nurses can give patients professional and standardized health education, as well as disease information and emotional support, and can also help to stabilize patients' emotions from 2 aspects. Nurses develop a relationship with patients in the role of relatives, which can reduce unfamiliarity and obtain a better nursing effect.

Clinical nursing work can be tedious, which may forget or misoperate by nurses. Mind mapping can effectively 
Table 5 Comparison of complications incidence between the 2 groups [n (\%)]

\begin{tabular}{lccccc}
\hline Group & No. & Hemorrhage & Subcutaneous effusion & Upper limb swelling & Skin flap necrosis \\
\hline Observation group & 128 & $17(13.28)$ & $7(5.47)$ & $14(10.94)$ & $3(2.34)$ \\
Control group & 128 & $25(19.53)$ & $11(8.59)$ & $22(17.19$ & $8(6.25$ \\
$\chi^{2}$ & & 1.823 & 0.956 & 2.069 & 2.375 \\
$P$ & & 0.177 & 0.328 & 0.150 & 0.123 \\
\hline
\end{tabular}

simplify necessary information, intuitively demonstrate the clinical nursing path, and assist nurses in sorting out their ideas for clinical nursing work. Furthermore, mind mapping can help to organize nursing work, and to analyze and summarize the nursing plan, patients' needs, and common postoperative complications, providing effective reminders of the nursing priorities of breast cancer patients in the perioperative period and thus improving the quality of nursing to benefit patients (13).

Surgery and disease result in different degrees of psychological stress. This state of tension and anxiety causes nerve impulses, which can be transmitted to the hypothalamus through the brainstem to promote the secretion of corticotropin-releasing factor (CRF). CRF can promote the synthesis and secretion of ACTH, which in turn stimulates the release of adrenocortical hormone. This can trigger a series of tense physiological and psychological reactions, including an increased heart rate and decreased skin temperature, which is not conducive to a smooth operation. The present study showed that the levels of anxiety, depression, and physiological stress before the operation in the observation group were significantly lower than those in the control group. This result suggests that the "playing a relative role" whole-process escort method combined with mind mapping can effectively relieve the psychological and physical stress experienced by patients undergoing breast cancer surgery. The reason for the alleviation of the physical and mental stress seen with this approach may be that staff pretending to be relative can reduce patients' sense of unfamiliarity with the hospital environment and their fear of surgery. Also, this approach can help patients to have a better psychological state to face the operation, enhance compliance, improve the operative effect, and promote rehabilitation in the process of surgical treatment. Furthermore, the degree of fatigue in the observation group was significantly lower than that in the control group, while the sleep quality in the observation group was significantly higher than that in the control group. These results may be explained by a good psychological state having a positive effect on fatigue and sleep.

A series of nursing intervention processes are formulated through mind mapping, which can not only improve the quality of nursing. but can also improve patients' understanding of breast cancer treatment and nursing. Furthermore, it can eliminate the fear of disease and surgery, and establish good compliance. Moreover, it contributes to acceptance of and active participation in relevant recovery training, enhances self-care awareness and efficacy, and is conducive to the control of complications and disease recovery (14). Mind mapping is also convenient for nurses to accurately judge the occurrence of postoperative complications in patients with breast cancer. It contributes to the implementation of targeted nursing intervention measures for various complications, the effective control of complications, and summary and analysis of the experience. Moreover, it is beneficial to improving plans to control the occurrence of complications. In this study, no significant difference was found in the incidence of complications between the observation and control groups, which may be due to the incidence of complications in the 2 groups being low. Thus, it is necessary to increase the sample size for further exploration in future clinical research.

Generally speaking, the assignment of a whole-process escort playing a relative role combined with mind mapping has beneficial effects for patients with breast cancer. It can effectively improve the psychological state, degree of fatigue, and sleep quality of patients, and thus, is beneficial to their prognosis.

\section{Acknowledgments}

Funding: None. 


\section{Footnote}

Reporting Checklist: The authors have completed the CONSORT reporting checklist. Available at https://dx.doi. org/10.21037/apm-21-915

Trial Protocol: Available at https://dx.doi.org/10.21037/apm21-915

Data Sharing Statement: Available at https://dx.doi. org/10.21037/apm-21-915

Conflicts of Interest: All authors have completed the ICMJE uniform disclosure form (available at https://dx.doi. org/10.21037/apm-21-915). The authors have no conflicts of interest to declare.

Ethical Statement: The authors are accountable for all aspects of the work in ensuring that questions related to the accuracy or integrity of any part of the work are appropriately investigated and resolved. The study was approved by ethics board of Wuhan Children's Hospital (Wuhan Maternal and Child Healthcare Hospital, No. 2021R55-E01). All procedures performed in this study involving human participants were in accordance with the Declaration of Helsinki (as revised in 2013). Participants' informed consent was obtained.

Open Access Statement: This is an Open Access article distributed in accordance with the Creative Commons Attribution-NonCommercial-NoDerivs 4.0 International License (CC BY-NC-ND 4.0), which permits the noncommercial replication and distribution of the article with the strict proviso that no changes or edits are made and the original work is properly cited (including links to both the formal publication through the relevant DOI and the license). See: https://creativecommons.org/licenses/by-ncnd $/ 4.0 /$.

\section{References}

1. Qaseem A, Lin JS, Mustafa RA, et al. Screening for Breast Cancer in Average-Risk Women: A Guidance Statement From the American College of Physicians. Ann Intern Med 2019;170:547-60.

2. El-Shennawy L, Dubrovskyi O, Kastrati I, et al.
Coactivation of Estrogen Receptor and IKK $\beta$ Induces a Dormant Metastatic Phenotype in ER-Positive Breast Cancer. Cancer Res 2018;78:974-84.

3. Deluche E, Leobon S, Desport JC, et al. Impact of body composition on outcome in patients with early breast cancer. Support Care Cancer 2018;26:861-8.

4. Heyland DK, Davidson J, Skrobik Y, et al. Improving partnerships with family members of ICU patients: study protocol for a randomized controlled trial. Trials 2018;19:3.

5. Spanoudis G, Demetriou A. Mapping Mind-Brain Development: Towards a Comprehensive Theory. J Intell 2020;8:19.

6. Wu HZ, Wu QT. Impact of mind mapping on the critical thinking ability of clinical nursing students and teaching application. J Int Med Res 2020;48:300060519893225.

7. Gale C, Glue P, Guaiana G, et al. Influence of covariates on heterogeneity in Hamilton Anxiety Scale ratings in placebo-controlled trials of benzodiazepines in generalized anxiety disorder: Systematic review and meta-analysis. J Psychopharmacol 2019;33:543-7.

8. Obeid S, Abi Elias Hallit C, Haddad C, et al. Validation of the Hamilton Depression Rating Scale (HDRS) and sociodemographic factors associated with Lebanese depressed patients. Encephale 2018;44:397-402.

9. Izgu N, Ozdemir L, Bugdayci Basal F. Effect of Aromatherapy Massage on Chemotherapy-Induced Peripheral Neuropathic Pain and Fatigue in Patients Receiving Oxaliplatin: An Open Label QuasiRandomized Controlled Pilot Study. Cancer Nurs 2019;42:139-47.

10. Dunleavy G, Bajpai R, Tonon AC, et al. Examining the Factor Structure of the Pittsburgh Sleep Quality Index in a Multi-Ethnic Working Population in Singapore. Int J Environ Res Public Health 2019;16:4590.

11. Bai XY, Li S, Wang M, et al. Krüppel-like factor 9 downregulates matrix metalloproteinase 9 transcription and suppresses human breast cancer invasion. Cancer Lett 2018;412:224-35.

12. Brinton LA, Brogan DR, Coates RJ, et al. Breast cancer risk among women under 55 years of age by joint effects of usage of oral contraceptives and hormone replacement therapy. Menopause 2018;25:1195-200.

13. Chen AP, Chang AH, Hsu EB. Using mind mapping 
technology for personal preparedness planning. Am J Disaster Med 2019;14:96-100.

14. Israel C, Pinto Zipp G, D'Abundo M, et al. Mind Mapping to Enhance Critical Thinking Skills in Physician Assistant
Education: A Randomized Controlled Study. J Allied Health 2020;49:135-40.

(English Language Editor: J. Reynolds)

Cite this article as: Fan L, Lei F, Zhu Z, Hu C, Ye L, Wang N. Nursing intervention using a whole-process escort playing a relative role combined with mind mapping in patients undergoing breast cancer surgery: a randomized trial. Ann Palliat Med 2021;10(12):12047-12054. doi: 10.21037/apm-21-915 